\title{
APPLICATIONS OF THE TUMURA-CLUNIE THEOREM
}

\author{
BY \\ CHUNG-CHUN YANG( $\left.{ }^{1}\right)$
}

\begin{abstract}
Some applications of the Tumura-Clunie theorem are given. Most of these concerned fixed points of compositions of entire functions.
\end{abstract}

I. Introduction. The purpose of this note is to develop some applications of the Tumura-Clunie theory (Hayman [2, p. 69]). It is assumed that the reader is familiar with the basic quantities of Nevanlinna Theory: $T(r, f), m(r, f), N(r, f), \ldots$ and their elementary properties.

II. Notation, terminology. 1. $f$ will always denote a nonconstant meromorphic function.

2. We shall denote by $S(r, f)$ any quantity satisfying $S(r, f)=o\{T(r, f)\}$, as $r \rightarrow+\infty$, possibly outside a set of $r$ of finite measure.

3. We shall denote by $a(z)\left(a_{1}(z), \ldots\right)$ meromorphic functions satisfying $T(r, a(z))=S(r, f)$.

4. By a differential polynomial $P(f)$ we understand a polynomial in $f(z)$ and its derivatives whose coefficients are of the form $a(z)$.

\section{Statement and applications of the Tumura-Clunie theorem.}

Theorem 1 (TUmura-Clunie [2]). Suppose $g(z)=f^{n}(z)+P_{n-1}(f)$, and that

$$
N(r, f)+N(r, 1 / g)=S(r, f) \text {. }
$$

Then $g(z)=h^{n}(z), h(z)=f(z)+a(z) / n$ and $h^{n-1}(z) a(z)$ is obtained by substituting $h(z)$ for $f(z), h^{\prime}(z)$ for $f^{\prime}(z)$, etc. in the terms of degree $n-1$ in $P_{n-1}(f)$.

REMARK. The conclusion still holds good if the condition (1) is replaced by:

$$
N(r, f)+N(r, 1 / g)=S_{0}(r, f),
$$

where $S_{0}(r, f)$ denotes any quantity which satisfies $S_{0}(r, f)=o(T(r, f))$ as $r \rightarrow+\infty$ through a set of $r$ of infinite measure.

We have

Received by the editors October 13, 1969 and, in revised form, February 10, 1970.

AMS Subject Classifications. Primary 3055, 3057.

Key Words and Phrases. Differential polynomial, fixed points.

( $\left.{ }^{1}\right)$ Most of this paper is based on the author's dissertation written under the guidance of Professor S. Hellerstein at the University of Wisconsin. 
Corollary (1-1). Let $A(z), B(z)$ be entire functions, $f(z)=e^{A(z)}, T(r, f)=$ $O\left(T\left(r, e^{B(z)}\right)\right)$. Then the identity

$$
a_{1}(z) e^{A(z)}+a_{2}(z) e^{B(z)}=a_{3}(z)
$$

cannot hold for an $a_{3}$ which is not identically equal to 0 .

Proof. The result follows immediately if $a_{1}(z) \equiv 0$ or $a_{2}(z) \equiv 0$. Therefore we may assume that both $a_{1}(z)$ and $a_{2}(z)$ are not identically equal to zero.

Multiplying both sides of (1-1) by $a_{1}(z)$, we have

$$
a_{1}^{2}(z) e^{A(z)}-a_{1}(z) a_{3}(z)=-a_{1}(z) a_{2}(z) e^{B(z)} .
$$

Setting $a_{1}(z) \exp [A(z) / 2]=H(z),-a_{1}(z) a_{2}(z) e^{B(z)}=g(z)$, we have

$$
H^{2}(z)-a_{1}(z) a_{3}(z)=g(z) .
$$

We note that

$$
\begin{aligned}
N(r, H)+N(r, 1 / g) & =N\left(r, a_{1}(z)\right)+N\left(r, 1 / a_{1}(z) a_{2}(z)\right) \\
& =S\left(r, e^{A(z)}\right)=S(r, H) .
\end{aligned}
$$

Therefore, the Tumura-Clunie theorem is applicable to the identity (1-3), and leads to

$$
H^{2}(z)=g(z) .
$$

This forces $a_{1}(z) a_{3}(z) \equiv 0$, therefore $a_{3}(z) \equiv 0$. In order to state Theorem 2, we need

Definition 1. To each function $\lambda(r)$, positive, continuous and nondecreasing on $0<r<+\infty$, we associate the class $F_{\lambda}$ of meromorphic functions $f$ satisfying $T(r, f)=O(\lambda(r))$, as $r \rightarrow \infty$ for all $r$ except a set of $r$ of finite measure. It is easily verified that $F_{\lambda}$ is a field.

THEOREM 2. Let $f$ be a meromorphic function with

$$
N(r, f)=S(r, f)
$$

satisfying

$$
f^{n}(z)+P_{n-1}(f)=b(z),
$$

where $P_{n-1}(f)$ is a differential polynomial in $f$ of degree at most $n-1$, and all the coefficients in $P_{n-1}(f)$ belong to $F_{\lambda}$ and $b(z) \in F_{\lambda}, b(z) \not \equiv 0$. Then $f \in F_{\lambda}$.

Proof. Assume the statement is false, i.e., $f \notin F_{\lambda}$. This implies that $T(r, b(z))$ $=S_{0}(r, f)$.

Combining this with the hypothesis on $N(r, f)$, we have

$$
N(r, f)+N(r, 1 / b(z))=S_{0}(r, f) .
$$

Therefore, Theorem 1 is applicable by the remark made after Theorem 1 . Thus we 
obtain $(f(z)+a(z))^{n}=b(z)$. Combined with $T(r, a(z))=S(r, f), T(r, b(z))=S_{0}(r, f)$ this leads to the contradiction $T(r, f)=S_{0}(r, f)$. Thus the theorem is proved.

A consequence of Theorem 2 is

Corollary (2-1) (Hellerstein AND Rubel [4]). $F_{\lambda}$ is algebraically closed in the field of all meromorphic functions.

The following theorem generalizes a result of Rosenbloom [3].

THEOREM 3. Let $f$ be a transcendental meromorphic function with $N(r, f)=S(r, f)$. Then $R(f)$ has infinitely many fixed points, where $R(z)=P(z) / Q(z)$, a rational function, and $P(z), Q(z)$ are two relative prime polynomials with degree of $P(z) \geqq 2$.

Proof. Assume $R(z)-z$ has finitely many fixed points. That is

$$
\frac{P(f)-z Q(f)}{Q(f)}
$$

has finitely many zeros.

Since $P(z), Q(z)$ are relatively prime, the zeros of $R(z)-z$ are just the zeros of $P(f)-z Q(f)$. Thus

$$
P(f)-z Q(f)=A(z) e^{B(z)},
$$

where $A(z)$ is a polynomial and $B(z)$ is an entire function. Consequently,

$$
\left(\alpha_{0} z+\beta_{0}\right) f^{n}(z)+\left(\alpha_{1} z+\beta_{1}\right) f^{n-1}(z)+\cdots+\left(\alpha_{n} z+\beta_{n}\right) f(z)+\alpha z+\beta=A(z) e^{B(z)}
$$

where $n$ is an integer $\geqq 2, \alpha_{i}(i=0,1,2, \ldots, n), \beta_{j}(j=0,1,2, \ldots), \alpha, \beta$ are constants and it is assumed that at least one of $\alpha_{0}, \beta_{0} \neq 0$.

According to the Tumura-Clunie theorem, we obtain that

$$
\left[f(z)+\frac{1}{n} \frac{\alpha_{1} z+\beta_{1}}{\alpha_{0} z+\beta_{0}}\right]^{n}=\frac{A(z) e^{B(z)}}{\alpha_{0} z+\beta_{0}} .
$$

Combining (3-3) and (3-4) we obtain

$$
R_{1}(z) f^{n-1}(z)+R_{2}(z) f^{n-2}(z)+\cdots+R_{n}(z) f(z) \equiv R_{0}(z),
$$

where $R_{i}(z), i=1,2, \ldots, n$, are rational functions and

$$
R_{0}(z)=\frac{\alpha z+\beta}{\alpha_{0} z+\beta_{0}}-\left(\frac{\alpha_{1} z+\beta_{1}}{\alpha_{0} z+\beta_{0}}\right)^{n} \not \equiv 0 .
$$

We conclude as before that

$$
(f(z)+R(z))^{n-1}=T(z),
$$

where $R(z), T(z)$ are rational functions. This is impossible because $f$ is transcendental and our proof is completed.

Along the same lines we have 
THEOREM 4. Let $f(z), g(z)$ be two transcendental entire functions and let $f(z)$ have finitely many fixed points. Let $A(z)$ be entire, and suppose that $A(z) \not \equiv g(z)-z$, and that $A(z)$ satisfies the condition

$$
T(r, A(z))=S(r, f) .
$$

(In the case $A(z) \equiv 0$ we also formally recognize that the condition (4-1) is fulfilled.) Then $f(g)(z)-A(z)$ has infinitley many fixed points.

Proof. Assume $f(g)(z)-A(z)$ has finitely many fixed points, that is

$$
f(g)(z)-A(z)-z=P_{1}(z) \exp \left[Q_{1}(z)\right],
$$

where $P_{2}(z)$ is a polynomial and $Q_{2}(z)$ is an entire function.

On the other hand, according to the hypothesis, we have

$$
f(z)-z=P_{2}(z) \exp \left[Q_{2}(z)\right],
$$

where $P_{2}(z)$ is a polynomial and $Q_{2}(z)$ is an entire function. Consequently,

$$
f(g)(z)-g(z)=P_{2}(g) \exp \left[Q_{2}(g)\right] .
$$

Combining (4-2) and (4-4) we obtain

$$
g(z)-z-A(z)=P_{1}(z) \exp \left[Q_{1}(z)\right]-P_{2}(g) \exp \left[Q_{2}(g(z))\right] .
$$

Since for any two entire transcendental functions $f$ and $g$

$$
T(r, f(g)) /\{T(r, f)+T(r, g)\} \rightarrow \infty
$$

(Hayman [2, pp. 50, 54]), we can apply Corollary (1-1) and conclude

$$
g(z)-z-A(z) \equiv 0 .
$$

This contradicts the hypothesis, so our proof is completed.

REMARK. $\left(f(z)=e^{z}+z\right.$ shows that the assumption $A(z) \not \equiv g(z)-z$ is crucial.) In the special case $A(z) \equiv 0$, we obtain another result of Rosenbloom [3].

\section{REFERENCES}

1. J. Clunie, On integral and meromorphic functions, J. London Math. Soc. 37 (1962), 17-27. MR 26 \#1456.

2. W. K. Hayman, Meromorphic functions, Oxford Mathematical Monographs, Clarendon Press, Oxford, 1964, pp. 68-73. MR 29 \#1337.

3. P. C. Rosenbloom, The fix-points of entire functions, Comm. Sém. Math. Univ. Lund 1952, Tome Supplémentaire, 186-192. MR 14, 546.

4. A. L. Rubel and S. Hellerstein, Subfields that are algebraically closed in the fields of all meromorphic functions, Technical Report No. 3, Appl. Math. and Statist. Labs., Stanford Univ., Calif., 1963.

5. Y. Tumura, On the extension of Borel's theorem and Saxer-Csillag's theorem, Proc. Phys. Math. Soc. Japan (3) 19 (1937), 29-35.

UNIVERSITY OF WISCONSIN, MAdison, Wisconsin 53706 